\title{
PERFORMANCE ANALYSIS OF GOLDEN CODE
}

\author{
A.S.Inamdar', Mavita Vatambe ${ }^{2}$, Hameed Miyan ${ }^{3}$ \\ ${ }^{1}$ Professor of E\&CE Dept, BKIT Bhalki \\ ${ }^{2}$ M.Tech Scholar (DCN) BKIT, Bhalki \\ ${ }^{3}$ PhD Scholar VTU Belgaum, India
}

\begin{abstract}
In this paper we have analyzed the performance of algebraic space time code in wireless MIMO (multiple input multiple output) system, OFDM is a unique kind of multicarrier transmission technique that divides the communication channel into several equally spaced frequency bands. This ASTC code is also called as golden code it has full rate and non vanishing constant minimum determinant for increasing spectral efficiency, this ASTC also has uniform transmitted energy per antenna, first we analyze the performance of golden code in correlated Rayleigh channel and we analyze the symbol error rate (SER), the simulated result shows that golden code has high SNR(signal to noise ration) and low BER(bit error rate) compare to alamouti scheme.
\end{abstract}

Keywords: MIMO, OFDM, ASTC code, Rayleigh Channel, spatial correlation, Bit Error Rate, Capacity.

\section{INTRODUCTION}

Orthogonal frequency division multiplexing (OFDM) is a unique kind of multicarrier transmission technique that divides the communication channel into several equally spaced frequency bands. The bit streams are divided into many sub streams and send the Information over different sub channels, each sub carrier is orthogonal with other sub carrier and it is carried out by a modulation scheme. OFDM is formed with two equalizers those are Minimum mean square error (MMSE) and zero forcing equalizer (ZFE),OFDM has several advantages like inter channel interference reduction, high security and better performance in multipath fading, equalizers are used to remove the inter symbol interference(ISI) generated from the transmitted data [1][2].

Multiple input multiple output (MIMO) is a multiple antenna system, In the MIMO technology multiple signals are transmitted into the wireless medium and multiple signals are received from the wireless medium to improve the wireless channel performance. The MIMO Transmission is extremely spectrum efficient technology that uses several antennas at both ends of the communication link. It has higher data rate greater range by increased number of users reliability can be enhanced by multiplying spectral efficiency. The MIMO takes advantage of multiple propagation to increase throughput range and reliability this is accomplished by sending and receiving more than one data signal in the same radio channel at the same time. the measure problem with the OFDM is PAPR(peak average power ratio) this problem will be solved by the ASTC encoder, To increase the spectral efficiency of the system we need to reduce the PAPR, it leads to better improvement in BER[3][6],

By providing a temporal and a spatial multiplexing modulation, the space time codes are used to improve
MIMO performances. The alamouti code[4] and the golden code[5] represents the most known codes and widely used codes, the golden codes has been proposed in 2004 for $2 * 2$ MIMO system it has full rate, full diversity space time code that has a maximal coding gain. Thanks to its algebraic construction

This paper shows that ASTC code outperforms the alamouti codes in Rayleigh channels. The ASTC codes lose their properties due to inter symbol interference (ISI), orthogonal frequency division multiplexing (OFDM) modulation can overcome this problem.

In this work, we propose a coded ASTC system in Rayleigh channel. And then we analyze the ASTC-MIMO-OFDM system in Rayleigh selective channel. We use a data aided channel estimation method based on the pilot symbol insertion in the detector to reduce the channel transfer function. Finally we analyze the system performances under spatially correlated channel.

\section{PROPOSED SYSTEM MODEL}

The block diagram of proposed system model is shown in fig, 1 we are considering the system model with two transmit antenna and two receive antenna, the generated binary sequence is given to the quadrature amplitude modulation after the modulation the possible output values of 4-QAM is $1+\mathrm{j}, 1-\mathrm{j},-1+\mathrm{j}$ and $-1-\mathrm{j}$. modulated signal is given to the golden encoder the encoded value is in matrix form shown in 1,the modulator is consist of IDWT block, after the channel MMSE estimator is used to check the error probability. 


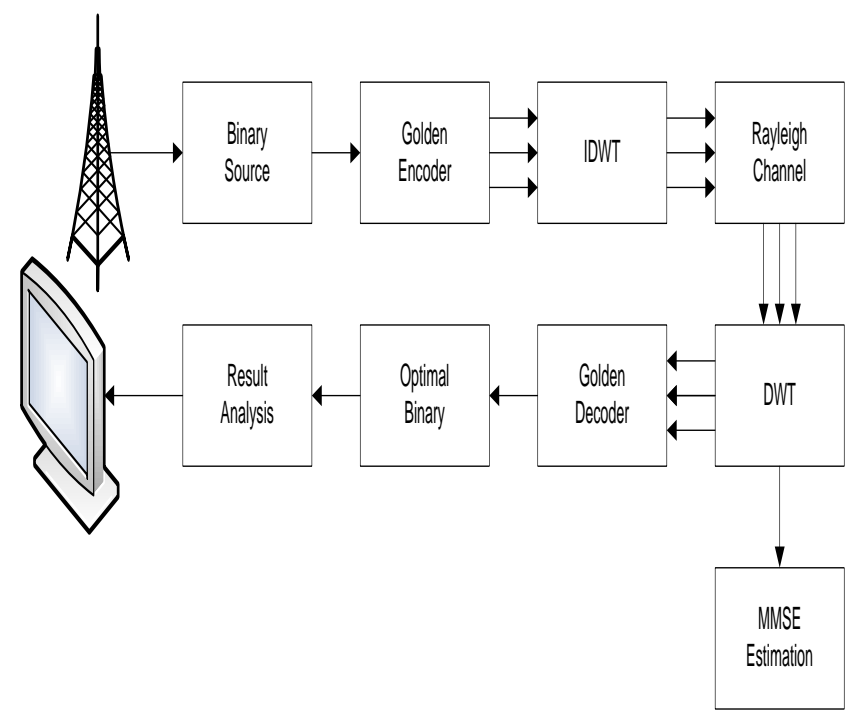

Fig 1: Block diagram of proposed system

These four information sequence are encoded by ASTC encoder

$$
C=\frac{1}{\sqrt{5}}\left(\begin{array}{ll}
\alpha(a+\theta b) & \alpha(c+\theta d) \\
\bar{\alpha}(c+\bar{\theta} d) & \bar{\alpha}(a+\bar{\theta} b)
\end{array}\right) \ldots . .1
$$

Where $\theta=\frac{1+\sqrt{5}}{2}=1.618$ (golden value),

$$
\bar{\theta}=\frac{1-\sqrt{5}}{2}, \alpha=1+i-\theta \text { and } \bar{\alpha}=1+i-i \bar{\alpha}
$$

a,b,c and d are modulated symbols.

The general transmission model of MIMO system is

$$
\mathrm{Y}=\mathrm{HX}+\mathrm{W}
$$

Where $\mathrm{X}$ is transmitted symbol $\mathrm{H}$ is channel matrix $\mathrm{W}$ is Gaussian noise and $\mathrm{Y}$ is output signal.

To have full rate square codes using QAM constellation, we consider square $2 * 2$ linear dispersion. we can express the code word $\mathrm{X}$ as the result of multiplication of each four consecutive symbols of information sequence $S$ by the matrix $\varphi_{t}$

$$
\varphi_{t}=\left[\begin{array}{llll}
\alpha & \alpha \theta & 0 & 0 \\
0 & 0 & \overline{i \alpha} & \overline{i \alpha \theta} \\
0 & 0 & \alpha & \alpha \theta \\
\bar{\alpha} & \overline{\alpha \theta} & 0 & 0
\end{array}\right]
$$

At time $(\mathrm{t}, \mathrm{t}+1)$, we can express the vector $X_{t}$ as shown below where first two lines are transmitted over antenna 1 , and the next two lines are transmitted over antenna 2.

$$
X_{t}=\left[\begin{array}{c}
(\alpha(a+\theta b))_{(t, 1)} \\
(\bar{\alpha}(c+\bar{\theta} d))_{(t+1,1)} \\
(\alpha(c+\theta b))_{(t, 2)} \\
(\bar{\alpha}(a+\bar{\theta} b))_{(t+1,2)}
\end{array}\right]
$$

\subsection{Channel Model}

In this proposed system we are using frequency selective Rayleigh channel and the channel taps remain constant during data transmission. The channel impulse response between $q^{\text {th }}$ transmitting antenna and $p^{\text {th }}$ receiving antenna is given by[7]

$$
h_{k}^{p, q}=\sum_{l=0}^{L-1} h_{k}^{p, q}(l) \delta(k-l)
$$

Where $h_{k}^{p, q}(l)$ is the $1^{\text {th }}$ path from the $q^{\text {th }}$ transmitting antenna to $p^{\text {th }}$ receiving antenna at time $\mathrm{K}$ and $\mathrm{L}$ is the largest order among all impulse responses. The channel taps sequence $\left\{h_{k}^{p, q}(l)\right\}$ is a correlated complex Gaussian process with zero mean and the same variance $\sigma_{h}^{2}$ and the autocorrelation function is given by

$$
\mathrm{E}\left\{h_{k}^{p, q}(l)\left[h_{k-k}^{m, n},(l)\right]\right\}=\rho_{R x}^{(m, p)} \rho_{T x}^{(n, q)} J_{0}\left(2 \pi f_{m} k^{\prime}\right) \delta\left(l-l^{\prime}\right)
$$

Where $f_{m}$ is normalized Doppler shift, $J_{0}$ is the Bessel function with zero order, $\rho_{R x}^{(m, p)}, \rho_{T x}^{(n, q)}$ refers respectively to the correlation coefficient between the received antenna $(\mathrm{m}, \mathrm{p})$ and the transmitted antenna $(\mathrm{n}, \mathrm{q})$ to obtain a correlated Rayleigh fading channel, the autocorrelation function of $\left\{h_{k}^{p, q}(l)\right\}$ process is given by

$$
R_{h}=\sigma_{h}^{2} \exp \left(j 2 \pi f k_{c}\right) j_{0}\left(2 \pi f_{m} k\right)
$$

\subsection{Sphere Decoding Algorithm}

The principle of sphere decoding algorithm is to search the closest constellation point to the received signal with in a sphere of some initial radius. If a point is found and if the distance between the centre and the point is less than the radius. The radius is updated to that distance and the process is continued till only one point is left in the sphere. That will be the closest constellation point to the received point. If a point is not found initially, then the sphere radius is incremented and the same process is followed.

\section{RESULT AND DISCUSSION}

The fig. 2 shows the symbol error rate(SER) performance of golden code for two transmit and two receive antenna using sphere decoder. And it has been obtained that the SER will remain constant and lies between $10^{-6} \& 10^{-7}$ for different signal to noise ratio(SNR), i.e. varies from 0 to $25 \mathrm{db}$.there result may leads to deals with real channel conditions mainly if we use Rayleigh channel with unknown channel coefficient. 


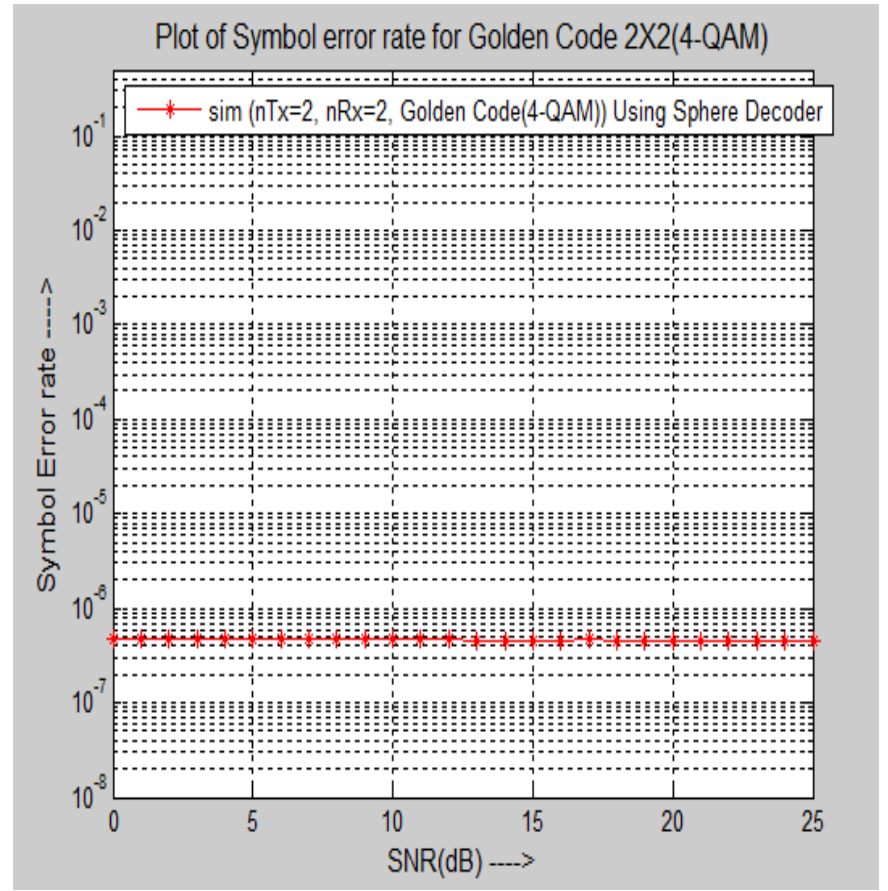

Fig 2: simulated result of golden code

\section{CONCLUSION}

We proposed a MIMO transmission system, based on Algebraic space time coding which has good properties. Simulated result show that the golden code has reasonable BER that outperforms the classical alamouti code over correlated Rayleigh fading channel. in realistic multipath channel, frequency selectivity can be solved by the use of OFDM modulation. numerical results show that ASTC codes maintain their properties and achieve good BER performances compare to the classical alamouti MIMOOFDM system.

\section{REFERENCES}

[1]. K.Thilagam1 and K.Jayanthi "A low Complex modified golden Code in a cooperative relaying System" International Journal of Computer Networks \& Communications (IJCNC) January (2014)

[2]. Anupama Sanyal, Parminder Singh "BER Improvement in OFDM with ZF and MMSE Equalizers Using ASTC Encoder in Different fading channels" International Journal of Advanced Engineering Research and Science (IJAERS) june(2014)

[3]. D. Meenakshi, S. Prabha, N. R. Raajan "Evaluation of BER for various Fading channel in dwt based MIMOOFDM system" International Journal of Engineering and Technology (IJET) May(2013).

[4]. S. M. Alamouti, "A simple transmit diversity technique for wireless communications," IEEE J. Select. Areas Commun., vol. 16, pp. 1451 1458, Oct. (1998).

[5]. J. C. Belfiore, G. Rekaya, and E. Viterbo, "The golden code: a 2 × 2 fullrate space-time code with non-vanishing determinants," in Information Theory, 2004. ISIT 2004. Proceedings. International Symposium on, Jun./Jul. 2004, pp. 310-310.
[6]. Kamna Mishra, Prof. Divya Jain and Prof. Vikas Gupta "Adaptation of Golden Codes with a Correlated Rayleigh Frequency- Selective Channel in OFDM System with Imperfect Channel Estimation" International Journal of Electrical, Electronics and Computer Engineering September (2012)

[7]. Ines BEN HASSINE, Ridha BOUALLEGUE "Performance analysis of algebraic space time code under correlated and uncorrelated channels" (2012)

[8]. Zhang.W, X. Gen and K.B. Letaief, "Spacetime/frequency coding for MIMO-OFDM in next generation broadband wireless systems", Proc.IEEE Wireless Commun., 2007.

[9]. Bannour, M. Ammari, S. Y, and R. Bouallegue, "On the capacity of ASTC-MIMO-OFDM system in a correlated Rayleigh frequencyselective channel," IEEE Veh. Technol. Conf., 2011. 